\title{
O uso da eletrotermofototerapia em pacientes com disfunções temporomandibulares
}

\author{
The use of electrothermophototherapy in patients with temporomandibular disorders \\ El uso de electrotermofototerapia en pacientes con trastornos temporomandibulares
}

Recebido: 19/12/2021 | Revisado: 27/12/2021 | Aceito: 01/01/2022 | Publicado: 02/01/2022

\author{
Fábio Teixeira Monteiro \\ ORCID: https://orcid.org/0000-0003-2041-2339 \\ Centro Universitário Mário Pontes Jucá, Brasil \\ E-mail: fabiot.monteiro@gmail.com \\ Rafaelly Oliveira Alves \\ ORCID: https://orcid.org/0000-0002-2054-2881 \\ Centro Universitário Mário Pontes Jucá, Brasil \\ E-mail: rafaellyoliveira10@ hotmail.com \\ Thais Ferreira Campello Diniz \\ ORCID: https://orcid.org/0000-0002-0437-745X \\ Centro Universitário Mário Pontes Jucá, Brasil \\ E-mail: campellothais69@gmail.com \\ Thauan Narciso de Lima Ferro \\ ORCID: https://orcid.org/0000-0002-0174-6467 \\ Centro Universitário Mário Pontes Jucá, Brasil \\ E-mail: thauan.ferro@umj.edu.br
}

\begin{abstract}
Resumo
A articulação temporomandibular (ATM), é uma das articulações mais complexas do corpo humano, indispensável e essencial sua biomecânica e suas funções são importantíssimas para as atividades diárias do ser humano, deglutição, fala, respiração, mastigação entre outras. Infelizmente na atualidade desordens nessa articulação vem acometendo um grande contingente populacional, não se limitando a faixas etárias. A evolução da fisioterapia tem se mostrado cada vez mais evidente e constante é de suma importância no tratamento dessas desordens, possibilitando não somente o protocolo de tratamento convencional, mas recursos eletrotermofoterapêuticos que auxiliam na busca pelo o alívio dos sintomas. O objetivo desta pesquisa foi verificar o uso da eletrotermofototerapia em pacientes com disfunções temporomandibulares. Trata-se de uma revisão literária realizada nas bases de dados Scielo Lilacs e Pubmed. Os estudos apontaram que o uso dos recursos é benéfico e eficaz. Conclui-se que os recursos utilizados alinhados ao tratamento convencional provêm melhorias no alívio da sintomatologia, mas ainda há uma escassez de literatura e estudos sobre o tema.
\end{abstract}

Palavras-chave: Eletrotermofototerapia; Disfunções; Pacientes.

\begin{abstract}
The temporomandibular joint (TMJ) is one of the most complex joints in the human body, its biomechanics essential and essential, and its functions are extremely important for human daily activities, swallowing, speaking, breathing, chewing, among others. Unfortunately, currently, disorders in this articulation have affected a large population, not limited to age groups. The evolution of physiotherapy has been shown to be more and more evident and constant is of paramount importance in the treatment of these disorders, enabling not only the conventional treatment protocol, but electrothermophototherapeutic resources that help in the search for symptom relief. The aim of this research was to verify the use of electrothermophototherapy in patients with temporomandibular disorders. This is a literary review carried out in the Scielo Lilacs and Pubmed databases. Studies have shown that the use of resources is beneficial and effective. It is concluded that the resources used in line with conventional treatment provide improvements in symptom relief, but there is still a shortage of literature and studies on the subject.
\end{abstract}

Keywords: Electrothermophototherapy; Dysfunctions; Patients.

\section{Resumen}

La articulación temporomandibular (ATM) es una de las articulaciones más complejas del cuerpo humano, su biomecánica esencial y esencial, y sus funciones son sumamente importantes para las actividades diarias del ser humano, tragar, hablar, respirar, masticar, entre otras. Desafortunadamente, en la actualidad, los trastornos en esta articulación han estado afectando a una gran población, no limitándose a grupos de edad. La evolución de la fisioterapia se ha demostrado cada vez más evidente y constante es de suma importancia en el tratamiento de estos trastornos, habilitando no solo el protocolo de tratamiento convencional, sino los recursos electrotermofototerapéuticos que ayudan en la búsqueda del alivio de los síntomas. El objetivo de esta investigación fue verificar el uso de electrotermofototerapia en pacientes con trastornos temporomandibulares. Se trata de una 
revisión literaria realizada en las bases de datos Scielo Lilacs y Pubmed. Los estudios han demostrado que el uso de recursos es beneficioso y eficaz. Se concluye que los recursos utilizados en consonancia con el tratamiento convencional aportan mejoras en el alivio de los síntomas, pero aún existe escasez de literatura y estudios sobre el tema.

Palabras clave: Electrotermofototerapia; Disfunciones; Pacientes.

\section{Introdução}

A articulação temporomandibular (ATM) é uma articulação sinovial. Articulação sinovial tem por definição a presença da sinóvia ou líquido sinovial. Este possibilita à articulação a realização de amplos movimentos em diferentes eixos. A composição anatômica da articulação sinovial é feita invariavelmente por: peças ósseas, cartilagem articular, cápsula articular, cavidade articular discos e ligamentos (Santos, 2010).

A ATM, classificada como a mais complexa do organismo humano, pode ser acometida pelas mesmas doenças e desordens que afetam outras articulações do sistema músculo-esquelético, como os deslocamentos de disco, doenças articulares degenerativas (osteoartrite), artrites inflamatórias e sinovites (Ramos et al. 2003).

A etiologia das disfunções temporomandibulares (DTMs) continua a ser amplamente estudada e a proposta atualmente mais consensual é que seja multifatorial. Os possíveis fatores englobam: stress emocional, ansiedade, distúrbios de interferência do disco, incongruências das estruturas articulares, trauma, hipermobilidade da ATM, hiperatividade muscular mastigatória e cervical, alterações posturais, prematuridades e interferências oclusais, alteração da posição e/ou perda de dentes e hábitos parafuncionais (Almeida et al. 2013).

Amoras et al. (2019) afirmam que a DTM é definida por um conjunto de desordens na articulação temporomandibular, sendo classificada em três tipos: Muscular, articular e degenerativa acometendo $70 \%$ das mulheres, a disfunção tem relação direta com a ATM e com suas partes constituintes músculos, disco articular e seus ligamentos, copiosas e distintas podem ser as suas causas. Ainda pouco conhecida entre os próprios fisioterapeutas a área vem ganhando espaço no tratamento do conjunto dessas desordens, seus sintomas dificultam a realização de atividades diárias simples e essenciais para uma boa qualidade de vida.

Segundo Cruz et al. (2020) é possível encontrar como sinais e sintomas de DTM as: Cefaleias, otalgias, mialgias, ruídos articulares, tonturas, desvios mandibulares, dor na ATM, entre outras desordens do sistema estomatognático e sistêmicas. Por ela ser uma patologia de múltiplos fatores, requer um tratamento baseado em diagnóstico correto, estabelecido a partir de informações sobre possíveis fontes causadoras, por meio do levantamento de sinais e sintomas para cada paciente de maneira individual.

O sistema estomatognático é responsável pelos movimentos de ingestão e mastigação, bem como para um fala, deglutição e Respiração. Estes movimentos são finamente controlados e força variada. Façam o desequilíbrio do aparelho mastigatório pode repercutir no sistema tônico postural, levando a modificações corporais importantes para diversas faixas etárias. Entretanto, estas mudanças são distúrbios anatomofisiológicos que se manifestam geralmente na infância, pois é neste período que o corpo está se desenvolvendo e sujeito uma Como aspectos psicossociais, que deu certo na biomecânica destes (Simões et al. 2015).

Segundo Bellomo JR (2014), em meados de 1983, o que já tinha sido nomeado de diversas outras formas, síndromes, distúrbios entre outros, foi concretizado e perpetuado pela Associação Dental Americana (ADA), que tornou consolidada e tomou como verdade absoluta a definição que Bell trouxe em 1982: Desordens temporomandibulares.

Desafortunadamente como o termo não é muito insigne, a uma grande porcentagem populacional que retém, porém não sabe que a possui, mas a procura prévia pelo profissional idôneo de fisioterapia, adjunto a uma equipe multidisciplinar pode interferir positivamente em seu prognóstico a fisioterapia possibilita não somente o tratamento convencional como 
abundantes recursos terapêuticos incluindo a eletrotermofoterapia. (Cavalcante et al. 2020).

Para Grossman et al. (2012) pesquisadores afirmam com clareza que concordam que deve haver cautela na recomendação de tratamentos invasivos e irreversíveis, principalmente no manuseio inicial das DTMs. Há uma série de terapias não invasivas e reversíveis que, nesses casos, úteis no controle da dor da maioria dos pacientes com essa disfunção, particularmente as funcionais ou idiopáticas.

Sendo afluente entre os meios para reabilitação o recurso por usufruir de meios térmicos, eflúvio luminoso e correntes elétricas vem coadjuvando excelentes resultados junto ao tratamento convencional das DTMs, a eletrotermofoterapia vem sendo um notável e perceptível aliado no alívio da sintomatologia (Damasceno \& Barbieri, 2014).

Sucintamente para assolar ou suavizar um possível quadro álgico consequente da disfunção, por meio de eletrodos o tratamento que tem como base, correntes elétricas, interpõe-se no ato de transmissão de sinais ao cérebro, ou promove contração elétrica no musculo almejado a depender do caso, intensidade, potência ou do aparelho utilizado. A boa notícia é que estes aparelhos não só promovem contração muscular e tem função de analgesia como promove relaxamento muscular, cicatrização, ainda podendo atuar na regeneração de determinados tecidos (Souza \& Meija, https://portalbiocursos.com.br).

Ultrassom, neuroestimulação elétrica transcutânea (TENS) e laser são arquétipos de recursos logrados amiudadamente no tratamento das desordens, este artigo trata-se de uma revisão literária que tem como cardinal propósito analisar o uso desse recurso no tratamento de pacientes que são acometidos por DTMs (Goulart et al. 2018).

A presente revisão literária se propõe a investigar o uso da eletrotermofoterapia em pacientes que sofrem com a disfunção temporomandibular, essa revisão se faz necessária devido a eficácia no alívio da sintomatologia que esses recursos proporcionam. A área ainda é carente de pesquisas mais aprofundadas, todavia, um dos objetivos desse estudo é de ampliar o conhecimento dessa área.

\section{Metodologia}

$\mathrm{O}$ universo da pesquisa utilizado foi o referencial bibliográfico, com autores que discutem o uso da eletrotermofoterapia em pacientes que sofrem com a disfunção temporomandibular. Levando em consideração o objeto a ser estudado, esta pesquisa foi de abordagem qualitativa.

Existem diferentes formas de se realizar uma revisão da literatura. Elas baseiam-se desde em técnicas como a revisão bibliográfica tradicional, também conhecida como revisão narrativa, alicerçada no uso de métodos específicos que visam a busca de um determinado assunto em acervos da literatura, até no uso de mecanismos e metodologias utilizados por acadêmicos e pesquisadores nos campos da saúde e educação para descrever o estado da arte de um tema (Botelho et.al. 2011).

Assim, o referido artigo teve seu processo de formação no período de três meses (outubro, novembro e dezembro) do ano de 2021. Tendo como bases de dados os sites científicos Scielo, Lilacs e Pubmed, selecionando-se artigos dos últimos cinco anos.

Durante a pesquisa foram encontrados 50 artigos que abordavam a temática, apenas 15 artigos mais recentes foram incluídos nessa revisão bibliográfica utilizando os descritores "eletrotermofoterapia AND pacientes AND disfunções temporomandibulares OR desordens temporomandibulares", Foram descartadas pesquisas que não estavam relacionados ao objeto de estudo.

Os artigos selecionados na pesquisa estão relacionados em fluxograma abaixo (Figura 1). 
Figura 1: processo de identificação e inclusão dos estudos.

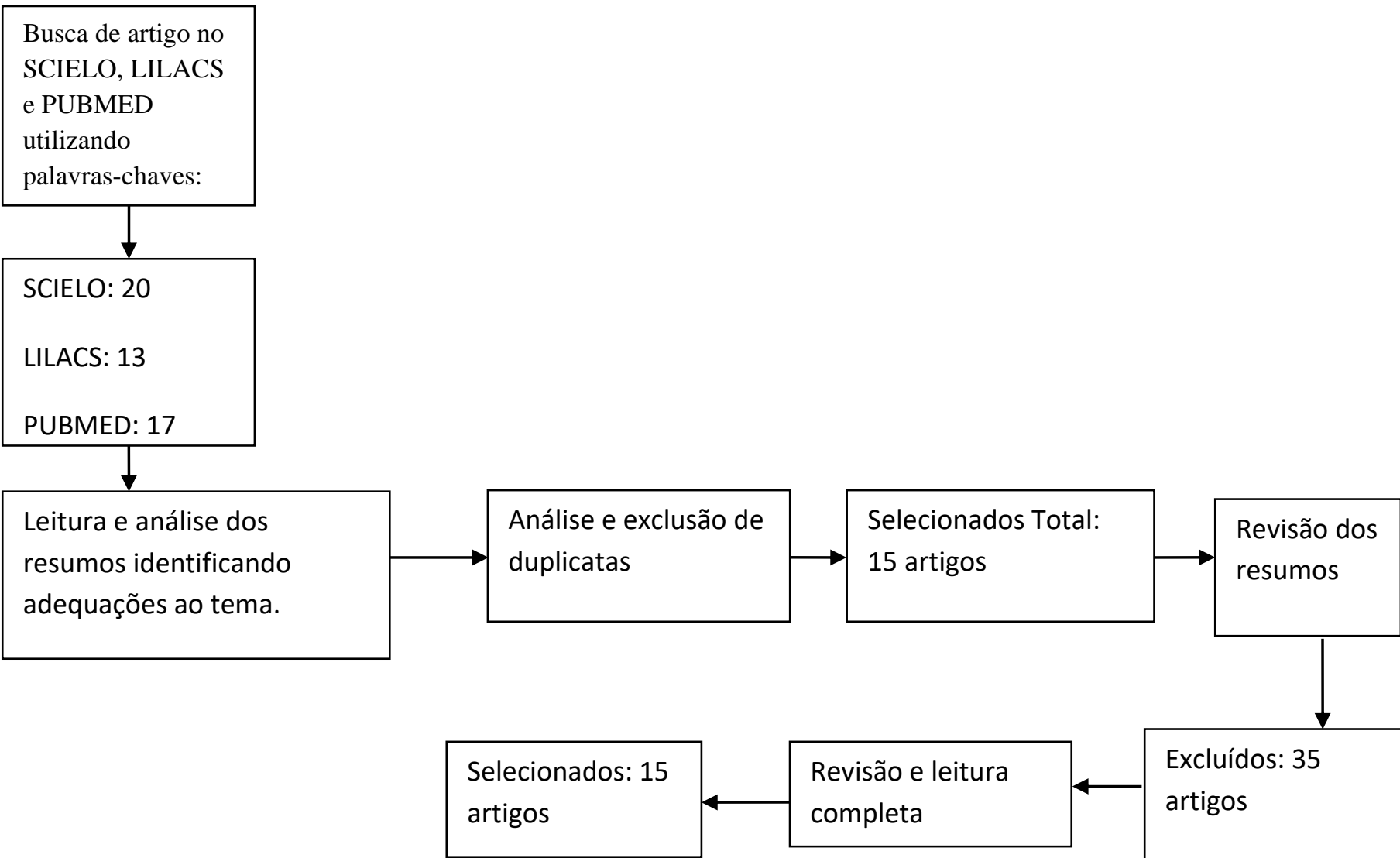

Fonte: Autores.

\section{Resultados e Discussão}

O quadro a seguir faz uma síntese comparativa entre artigos e autores relacionado ao uso da eletrotermofoterapia em pacientes com disfunções temporomandibulares. 
Quadro 1: Artigos selecionados.

\begin{tabular}{|c|c|c|}
\hline AUTOR & $\begin{array}{c}\text { TÍTULO } \\
\end{array}$ & ANO \\
\hline $\begin{array}{l}\text { DAMASCENO, F. M. e } \\
\text { BARBIERI, L. G. }\end{array}$ & $\begin{array}{l}\text { O tratamento fisioterapeutico nas disfunções } \\
\text { temporomandibular: uma revisão integrativa . }\end{array}$ & 2014 \\
\hline CAVALCANTE et.al. & $\begin{array}{l}\text { Abordagem terapeutica multidisciplinar para o } \\
\text { tratamento de dores orofaciais: uma revisão de } \\
\text { literatura. }\end{array}$ & 2020 \\
\hline $\begin{array}{l}\text { SOUZA, T. M. A. de e MEIJA, D. } \\
\text { P. M. }\end{array}$ & $\begin{array}{c}\text { A utilização da estimulação elétrica nervosa } \\
\text { transcultanea (tens) no tratamento da sindrome } \\
\text { miofascial doloroso }\end{array}$ & $\begin{array}{ll}---- \\
-1\end{array}$ \\
\hline BELLOMO jr. & $\begin{array}{c}\text { Mandibular movements patterns in the } \\
\text { temporomandibular disorders by kinematics } \\
\text { parameters }\end{array}$ & 2014 \\
\hline PINHEIRO M. & $\begin{array}{c}\text { - Ultrassom na fisioterapia para que serve e como } \\
\text { usar corretamente - tua saúde - } \\
\text { https://www.tuasaude.com/fisioterapia-com- } \\
\text { ultrassom }\end{array}$ & 2020 \\
\hline SILVA D.F.; MEJIA D.P.M & $\begin{array}{l}\text { Uso do tens na dor da disfunção } \\
\text { temporomandibular na fisioterapia. }\end{array}$ & 2014 \\
\hline $\begin{array}{l}\text { SOUZA A.C.O.C.; PEREIRA } \\
\text { P.C.; JUNIOR J.D.C }\end{array}$ & $\begin{array}{l}\text { A influência da laserterapia de baixa potência e do } \\
\text { ultrassom terapêutico na abertura da boca em } \\
\text { pacientes com disfunção temporomandibular, }\end{array}$ & 2020 \\
\hline $\begin{array}{l}\text { SCHROEDERL C.; CUNALI } \\
\text { R.S.; BONOTTO D.; FARIAS } \\
\text { A.C.; CUNALI P.A } \\
\end{array}$ & $\begin{array}{l}\text { Ultrassom, laser, e eletroterapias no tratamento da } \\
\text { dor por disfunção temporomandibular: revisão } \\
\text { sistemática. }\end{array}$ & 2013 \\
\hline AMORAS et.al. & $\begin{array}{l}\text { Efeitos de um protocolo fisioterapêutico para } \\
\text { tratamento de paciente com disfunção } \\
\text { temporomandibular }\end{array}$ & 2021 \\
\hline $\begin{array}{l}\text { GROSSMANN E.; TAMBARA } \\
\text { J.S.; GROSSMANN T.K.; } \\
\text { SIQUEIRA J.T.T }\end{array}$ & $\begin{array}{l}\text { O uso da estimulação elétrica nervosa } \\
\text { transcutânea na disfunção temporomandibular }\end{array}$ & 2012 \\
\hline $\begin{array}{l}\text { RODRIGUES-BIGATON, D; } \\
\text { ALMEIDA, A. F. N.; BERNI, K. } \\
\text { C. S.; PEDRONI, CR; } \\
\text { GONÇALVES, R. N; BÉRZIN, }\end{array}$ & $\begin{array}{c}\text { Utilização de diferentes estimulações elétricas } \\
\text { para o tratamento da dor em mulheres com } \\
\text { disfunção temporomandibular }\end{array}$ & 2008 \\
\hline $\begin{array}{l}\text { ASSIS, T. de O.; SOARES, M. dos } \\
\text { S.; VICTOR, M. M. }\end{array}$ & $\begin{array}{l}\text { O uso do laser na reabilitação das desordens } \\
\text { temporomandibulares }\end{array}$ & 2012 \\
\hline $\begin{array}{l}\text { MARTINS R. M. B. F.; SANTOS } \\
\text { M. H. S. M.; SILVA R. A.; } \\
\text { GONDIM N. F R. }\end{array}$ & $\begin{array}{l}\text { A resposta da Tens no controle da dor miofascial } \\
\text { dos pacientes portadores de disfunção } \\
\text { temporomandibular }\end{array}$ & 2004 \\
\hline PANHÓCA H. V. & $\begin{array}{c}\text { Laser e ultrassom combatem dor da disfunção } \\
\text { temporomandibular }\end{array}$ & 2018 \\
\hline GOULART, C. P. et al. & $\begin{array}{l}\text { Efeitos adversos da eletrotermofototerapia em } \\
\text { clínicas da cidade de Cascavel - PR. }\end{array}$ & 2018 \\
\hline $\begin{array}{l}\text { CRUZ J. H. A.; SOUSA L. X.; } \\
\text { OLIVEIRA B. F.; ANDRADE } \\
\text { JUNIOR F. P.; ALVES M. A. S. } \\
\text { G.; OLIVEIRA FILHO A. A }\end{array}$ & $\begin{array}{c}\text { Disfunção temporomandibular: revisão } \\
\text { sistematizada }\end{array}$ & ------ \\
\hline COSTA, G. DE A. & $\begin{array}{l}\text { Método para estimar a atenuação ultrassônica de } \\
\text { materiais utilizando a força de radiação }\end{array}$ & 2015 \\
\hline TACON et.al. & $\begin{array}{c}\text { A história da ultrassonografia no brasil e no } \\
\text { mundo }\end{array}$ & 2012 \\
\hline
\end{tabular}

Fonte: Autores.

A dor é uma disfunção que pode ser causada por trauma, onde tem que se observar detalhadamente e avaliada com cuidado todas as interações dos músculos da mandíbula, ATM, ossos do crânio, garganta, língua, dentes, enfim, todos os nervos e musculaturas que envolvem a cabeça e até mesmo a coluna e marcha, como observado no contexto do artigo. E despois de realizada toda essa avaliação facilita montar um bom protocolo de tratamento que não prejudique outras áreas do corpo (Silva \& Mejia, 2013).

Segundo Grossmann et al. (2012) uso da TENS em odontologia visa o controle da dor crônica, em casos selecionados 
e o relaxamento dos músculos mastigatórios. literaturas observaram que, em repouso, indivíduos com DTM muscular apresentam maior atividade mioelétrica dos músculos levantadores da mandíbula que o grupo controle, sendo mais evidente na porção anterior do músculo temporal. Quando empregaram a TENS, essa promoveu o alívio da dor com redução simultânea na atividade mioelétrica na porção anterior desse músculo em repouso.

Martins et al. (2004) relatam que a tens, Transcutaneous Eletrical Nerve Stimulation, é um recurso eletroterápico que tem sido recomendado no tratamento da DTM como um analgésico local. Seu efeito pode durar em média 72 horas, pois através da liberação de opiáceos endógenos a TENS ativa o mecanismo de controle ou centralmente, reduzindo a dor, consequentemente.

Um estudo observou que 95,3\% dos pacientes com dor facial idiopática persistente têm pelo menos um músculo com alteração do seu tônus em repouso, determinado pelo exame eletromiográfico. Após 45 minutos da TENS, esses valores voltaram para um padrão de normalidade. Esse mesmo estudo concluiu que o uso da TENS juntamente com o uso de dispositivos interoclusais leva a mandíbula para uma nova posição espacial. Essa nova oclusão determina um aumento significativo em qualidade e quantidade de força de mordida, revelando melhor eficiência da função muscular em, pelo menos, $25 \%$ ( $<$ < 0,05) para a maioria dos músculos avaliados, além da eficácia desse dispositivo na redução da dor (Grossmann et. al, 2012).

Desta forma, a TENS é um recurso fisioterápico que pode ser usado no controle da dor miofascial, comum às DTMs, e que pode melhorar a qualidade de vida dos pacientes portadores desta disfunção da ATM (Martins et al., 2004).

Segundo Silva e Meija (2014) os Parâmetros recomendados são:

Breve e intenso.

Frequência alta (abaixo de $100 \mathrm{~Hz}$ ).

Duração de 20 microssegundos (largo).

Amplitude forte ao nível de tolerância.

A fisioterapia com ultrassom é feita com o objetivo de aumentar o fluxo sanguíneo local e assim favorecer a cascata inflamatória, reduzindo o inchaço e estimulando as células inflamatórias, promovendo assim a cicatrização, remodelação do tecido e diminuindo o edema, dores e os espasmos musculares (Pinheiro, 2020).

É definido como uma forma de onda acústica inaudível, cujas frequências para tratamento por meio de vibrações mecânicas são superiores a $20.000 \mathrm{~Hz}$ (Borges, 2010).

De modo histórico em meados de 50, foi realizado o primeiro uso do ultrassom, desde então com o aumento da tecnologia o recurso sofreu diversas mudanças, melhorias, e não estacionou na evolução. Hoje existem duas modalidades para aplicação dele, contínuo ou pulsado (Tacon et. al., 2012).

Os Parâmetros recomendado são modo pulsado, Potência de 1,5w/cm³ e Frequência de 3 MHz. (Costa, 2015)

Vale ressaltar que é apenas uma sugestão, seu uso como o de qualquer outro recurso estará a depender de cada caso individualmente (Tacon et. al., 2012).

A respeito do uso do ultrassom no tratamento das disfunções temporomandibulares a literatura ainda não dispõe de materiais com evidência que permitam uma conclusão. A pesquisa relatada acima conseguiu mostrar melhora do grupo tratado com ultrassom em comparação com grupo tratado com placebo, mas ressalta que mais estudos são necessários para provar a sua eficácia (Schroeder et al., 2013).

Cabalmente utilizado em intensidade mais baixa, o laser é a amplificação de luz por emissão de radiação, possibilita melhorias desde o processo inflamatório, desfazendo edemas até uma analgesia. Está contraindicado na região ocular, ferimentos contaminados, gestantes, marcapassos e carcinomas. 
Protocolo sugerido para cicatrização: 1 a 3, com objetivo de analgesia: 1,3 a 4 e inflamação: 0,5 a 4.

Assis et al. (2012) inferem que o laser de baixa intensidade (LBI) tem se mostrado uma alternativa importante no alívio da dor e no restabelecimento da função, no que diz respeito ao tratamento das DTM. Por ser uma modalidade de tratamento não invasiva e de baixo custo, vem sendo frequentemente utilizada na prática clínica. Apesar das poucas evidências clínicas, de AsGaAl e AsGa mostraram ser eficazes no tratamento dos DTM na maioria dos estudos descritos. Todavia, a falta de consenso sobre os parâmetros utilizados é evidente e parece ser um fator limitante para conclusões mais acuradas a respeito do nível da eficácia do uso da LBI nesses casos.

Os autores sugerem que novos estudos sejam realizados, de preferência do tipo ensaios clínicos randomizados, e que parâmetros específicos sejam estudados e seus efeitos divulgados junto à comunidade científica e clínica a fim de otimizar os efeitos da LBI, sobretudo para que os pacientes que sofrem algum tipo de DTM possam se beneficiar, melhorando sua qualidade de vida (Assis et al. 2012).

Visando o alívio da dor hodiernamente tem sido bastante utilizada por diversos fisioterapeutas como protocolo a combinação de ultrassom e laser, embora ambos possuam o mesmo efeito fisiológico, a união promove melhores resultados (Panhóca, 2018).

Tanto o laser de baixa potência quanto o US terapêutico influenciam no aumento da abertura da boca de pacientes com DTM. Ao comparar as técnicas, foi possível observar que, proporcionalmente, o LBP reduziu mais o quadro álgico. Já o US proporcionou maior aumento da abertura bucal, embora haja o risco de causar dor no paciente se os parâmetros não forem controlados adequadamente. Ainda são necessários mais estudos com um maior número de pacientes, controlando a posição cervical na avaliação e no tratamento, além de aprofundar no entendimento das dores provocadas pelo uso do US (Souza et al. 2020).

\section{Considerações Finais}

Apesar de a fisioterapia possuir uma infinidade de técnicas alternativas, incluindo o próprio tratamento convencional, entretanto, há uma escassez de estudos sobre o uso da eletrotermofoterapia na terapêutica das desordens temporomandibulares. As recentes pesquisas confirmam que pacientes apresentam uma melhora significativa quando se acoplam os recursos de eletrotermofototerapia ao tratamento convencional ou quando ambos estão aliados. A tribulação é que as poucas investigações não permitem uma análise mais apurada da eficácia desses recursos terapêuticos. Sugere-se, portanto, a realização de estudos mais aprofundados nessa referida área.

\section{Referências}

Almeida, F., Pinho, J., Coimbra, D., Pais C., M. \& Ribeiro S. N. (2013). Biomecânica da articulação temporomandibular numa população de cantores. Revista Portuguesa de Estomatologia, Medicina Dentária e Cirurgia Maxilofacial. 54. 131-136. 10.1016/j.rpemd.2013.02.004

Amoras M. R., Lima P. R. R, Felix T.G. \& Freitas, M.T.M.W. (2021). Efeitos de um protocolo fisioterapêutico para tratamento de paciente com disfunção temporomandibular: um relato de caso. revista Inova saúde 11 (2).

Assis, T. O., Soares, M. S. \& Victor, M. M. (2012), Fisioterapia em Movimento 25 (2), 453 - 459

Bellomo Jr. D. P. (2014). 3D Mandibular movements patterns in the temporomandibular disorders by kinematics parameters. Doctoral Thesis- Faculty of Electrical Engineering of Federal University of Uberlândia. Uberlândia- Brazil

Botelho R. L. L, Cunha A. C. C., Macedo M. (2011), o método da revisão integrativa nos estudos organizacionais./revista eletrônica Gestão e sociedade 5(11) 121-136.

Braz. J. (2020). Of Develope. 6 (7). 44293-44310.

Cavalcante S. K. S., Linhares P. N., Couto A. F. P. M., Santiago F. T., Lima C. K. K., Pinto D. M. C. A., Bezerra V. Y., Mendes D. A. T. \& Dinelly P. M. E. (2020). Abordagem terapêutica multidisciplinar para o tratamento de dores orofaciais: uma revisão da literatura / revista Brazilian Journal of development 6 (7) 
Research, Society and Development, v. 11, n. 1, e8211124941, 2022

(CC BY 4.0) | ISSN 2525-3409 | DOI: http://dx.doi.org/10.33448/rsd-v11i1.24941

Costa, G. A. (2015). Método para estimar a atenuação ultrassônica de materiais utilizando a força de radiação. UFRJ/COPPE

Cruz J. H. A., Sousa L. X., Oliveira B. F., Andrade J. F. P., Alves M. A. S. G. \& Oliveira Filho A. A, (2020 ). Disfunção temporomandibular: revisão sistematizada, Arch Health Invest, 9 (6). 570-575

Damasceno, M. F. \& Barbieri, G. L. (2014). O tratamento fisioterapêutico nas disfunções temporomandibular: uma revisão integrativa . E F Deportes.com, Revista Digital. Buenos Aires, 18(188). http://www.efdeportes.com/

Goulart, C. P. (2018). Efeitos adversos da eletrotermofototerapia em clínicas da cidade de Cascavel - PR. Fisioterapia e Pesquisa. 25 (4) p. $382-387$. https://doi.org/10.1590/1809-2950/17001725042018

Grossmann E., Tambara J. S., Grossmann T. K. \& Siqueira Jr. T. T. (2012). O uso da estimulação elétrica nervosa transcutânea na disfunção temporomandibular Rev. Dor. São Paulo

Martins R. M. B. F., Santos M. H. S. M., Silva R. A. \& Gondim N. F. R (2004). A resposta da Tens no controle da dor miofascial dos pacientes portadores de disfunção temporomandibular. Fisioterapia Brasil 5 (4)

Panhóca H.V. (2018). Disfuncao-temporomandibular. www2.ifsc.usp.br/portal-ifsc/

Pinheiro M. (2020). Ultrassom na fisioterapia para que serve e como usar corretamente - Tua saúde - https://www.tuasaude.com/fisioterapia-com-ultrassom/

Ramos, A. C. A., Sarmenton A. V., Campos F. S. P. \& Gonzalez D. O. M. (2004). Articulação temporomandibular- aspectos normais e deslocamento de disco: imagem por ressonância magnética. Radio Bras 37(6): 449-454

Rodrigues, B. D., Almeida, A. F. N, Berni, K. C. S., Pedroni, C. R, Gonçalves, R. N \& Bérzin, F. (2008). Utilização de diferentes estimulações elétricas para o tratamento da dor em mulheres com disfunção temporomandibular. Brazilian Journal of Physical Therapy, 12 (6) 476 - 481

Santos, N. C. C. (2010). Articulação temporomandibular: anatomia, dinâmica e disfunções temporomandibulares. Trabalho de conclusão de curso (bacharelado - Odontologia) - Universidade Estadual Paulista, Faculdade de Odontologia de São José dos Campos, < http://hdl.handle.net/11449/121028 >

Souza, A. C. O. C., Pereira P. C. \& Junior J. D. C., (2020). A influência da laserterapia de baixa potência e do ultrassom terapêutico na abertura da boca em pacientes com disfunção temporomandibular. Fundação de Ensino e Pesquisa de Itajubá (FEPI). Revista Artigos.com

Schroeder, S. C., Cunali R. S., Bonotto F. A. C. \& Cunali P. A. R. A (2013). Ultrassom, laser, e eletroterapias no tratamento da dor por disfunção temporomandibular: revisão sistemática

Silva, D. F. \& Mejia D. P. M. Uso do tens na dor da disfunção temporomandibular na fisioterapia. Pós graduação em traumato ortopedia- Faculdade Ávila S $\mathrm{D}$

Simões, T. B., Abrahão, M. S., Coelho N. J. \& Sá, K. (2015). Efeito imediato da intervenção da atm na biomecânica da postura em escolares. Revista Pesquisa em Fisioterapia. 1. 10.17267/2238-2704rpf.v1i1.73

Tacon, B. C. K., Amaral N. W. \& Santos O. C. H. (2012). A história da ultrassonografia no Brasil e no mundo EFDeportes.com, Revista Digital.17(167): http://www.efdeportes.com/ 